\title{
Acido úrico y calciuria: valor pronóstico y diagnóstico en los trastornos hipertensivos de la gestación
}

\author{
Jorge Eduardo Vélez Arango*; Nubia Yolanda Chamorro Quiroz**; Carlos Miguel Céspedes Rubio***
}

\begin{abstract}
RESUMEN: En el presente estudio se analizan los niveles de calciuria y ácido úrico en un grupo de 135 pacientes con trastornos hipertensivos de la gestación que consultaron al servicio de admisiones obstétricas del Hospital de Caldas entre marzo $1^{\circ}$ y agosto 31 de 1994. En todas las pacientes se determinaron niveles de ácido úrico, creatinina, nitrógeno ureico, proteinuria en muestra ocasional, proteinuria en 24 horas, calciuria en 24 horas y recuento plaquetario. De acuerdo con el diagnóstico las pacientes se clasifican en: hipertensión inducida por el embarazo: $55,6 \%$, hipertensión arterial crónica: $5,5 \%$, hipertensión arterial crónica más preeclampsia sobreagregada: $11,9 \%$, hipertensión transitoria gestacional: $26,7 \%$. La edad promedio de las pacientes fue de 26,19 años $\pm 7,29$ años con una presencia de unión inestable del 32,6\%. El 54,1\% de ellas son primigestantes con una paridad promedio de 1,27 $\pm 1,93$ hijos.

Se comprueba una disminución estadísticamente significativa de los niveles de calciuria en las pacientes con hipertensión inducida por el embarazo, hipertensión arterial crónica + preeclampsia sobreagregada e hipertensión transitoria gestacional. Existe elevación significativa de los niveles de ácido úrico en las cuatro categorías de trastornos hipertensivos. Ambos hallazgos están de acuerdo con lo reportado por la literatura, razón por la cual se propone la determinación de la calciuria en orina de 24 horas como una prueba de diagnóstico y tamizaje de preeclampsia eclampsia.
\end{abstract}

PALABRAS ClAVES: preeclampsia - eclampsia. Calciuria en orina de 24 horas y ácido úrico como métodos diagnósticos de preeclampsia.

SUMMARY: The levels of calciuria and uric acid of a group of 135 patients with hipertensive disorders of the pregnancy who had consulted the admission service of the Caldas Hospital between the 1st. of March and the 31st. of August of 1994 were evaluated in this study. The patients were classified according to the diagnostic as follows: pregnancy induced hypertension (preeclampsia - eclampsia): $55,6 \%$; cronic arterial hypertension: $5,5 \%$; preeclampsia superimposed on chronic hypertension: $11,9 \%$; transient hypertension: $26,7 \%$. The levels of calciuria showed a significative stadistical reduction $(\mathrm{p}<0,01)$ in patients with pregnancy induced hypertension and preeclampsia superimposed on chronic hypertension. There was a significative increase of the levels of uric acid in all the hypertensive disorders. Both findings agree with that registered in the literature and confirm the potential use of the calciuria levels in 24 hours urine as a diagnostic and screening test in preeclampsia.

KEY WORDS: preeclampsia - eclampsia. Calciuria in 24 hours urine and uric acid as diagnostic methods of preeclampsia.

\section{Introducción}

Los trastornos hipertensivos de la gestación continúan representando una causa frecuente de morbimortalidad materna y perinatal. En nuestro medio, dicha patología es la primera causa de mortalidad materna (1).

A pesar de la gran cantidad de pruebas propuestas para el diagnóstico precoz de la enfermedad no se ha identificado aún la ideal (2).

Estudios recientes vienen señalando la importancia del metabolismo del calcio en la génesis y fisiopatología de la preeclampsia eclampsia $(3-6,8,10-11,16,23)$. Estos conceptos han conducido al desarrollo de una serie de investigaciones dirigidas a estimar los valores de calciuria en orina de 24 horas como método diagnóstico $(3-4,6,9-10)$. Taufield y colaboradores han comprobado

\footnotetext{
* Profesor Asistente, Jefe Sección Obstetricia, Departamento de Gineco-Obstetricia.

** Residente III, Departamento de Gineco-Obstetricia

*** $\quad$ Residente II, Departamento de Gineco-Obstetricia.
}

una menor excreción de calcio en orina de 24 horas en pacientes con preeclampsia o hipertensión crónica con preeclampsia sobreagregada. Tales estudios sugieren que la preeclampsia podría estar asociada con hipocalciuria secundaria al incremento de la reabsorción tubular de calcio (8). Se han considerado como valores normales para la excreción de calcio: $300+15 \mathrm{mg} / 24$ horas, considerándose como nivel crítico el de: $170+30 \mathrm{mg} / 24$ horas (26-27). El ácido úrico fue propuesto por Stander y colaboradores desde 1923 como una prueba predictiva de la aparición y severidad del síndrome. Lo que parece mostrar la experiencia clínica sinembargo, es que los niveles de ácido úrico presentan grandes variaciones cuando se comparan las cifras obtenidas en embarazadas normales y en pacientes con trastornos hipertensivos de la gestación en todas sus formas $(2,23)$.

El presente estudio intenta probar la utilidad en nuestro medio de las determinaciones de ácido úrico y calciuria en orina de 24 horas, como métodos diagnósticos de los trastornos hipertensivos de la gestación. 


\section{Material y métodos}

El presente es un estudio de tipo prospectivo analítico. Se incluyeron en la investigación todas las pacientes con trastornos hipertensivos de la gestación que consultaron al servicio de admisiones de obstetricia del Hospital de Caldas entre marzo $1^{\circ}$ y agosto 31 de 1994 . La información se consignó en un formulario precodificado diseñado para tal propósito. Todas las pacientes fueron hospitalizadas realizándose las siguientes pruebas de laboratorio: ácido úrico, nitrógeno ureico, urea, creatinina, recuento plaquetario, proteinuria y calciuria en orina de 24 horas. El ácido úrico y la calciuria en orina de 24 horas fueron determinados mediante la técnica de Wiener.

Se realizó seguimiento de todas las pacientes con el fin de identificar la evolución, tipo de parto, presencia de complicaciones maternas $y / 0$ fetales y establecer un diagnóstico final con base en los datos clínicos y de laboratorio disponibles.

Se utilizó para el manejo de la información un computador personal IBM compatible empleando el programa epidemiológico Epi Info, versión 5.01b (julio 1991). Se efectuaron análisis estadísticos descriptivos y se em- pleó la prueba estadística de $\mathrm{Z}$ para el análisis inferencial,(22) aceptándose un nivel de significancia del $1 \%(\mathrm{p}<0,01)$.

\section{Resultados}

Se incluyeron en la investigación 135 pacientes, las cuales se clasificaron según diagnóstico en: hipertensión inducida por el embarazo (preeclampsia - eclampsia) (HIE): 75 pacientes $(55,6 \%)$; hipertensión arterial crónica (HAC): 8 pacientes $(5,9 \%)$; hipertensión arterial crónica más preeclampsia sobreagregada (HAS): 16 pacientes (11,9\%); hipertensión transitoria gestacional (HTG): 36 pacientes $(26,7 \%)$.

La edad promedio de este grupo de pacientes es de $26,19 \pm 7,29$ años y un 32,6\% de ellas no tienen unión estable. El $54,1 \%$ son primigestantes, con una paridad promedio de 1,27 $\pm 1,93$ hijos para el grupo. E1 88,1\% de las pacientes asistió a control prenatal. La edad gestacional al momento de la consulta fue de $37,33 \pm 3,61$ semanas. La tabla 1 discrimina las variables edad, paridad, edad gestacional, tensión arterial sistólica, diastólica y media según diagnóstico.

Tabla 1

ACIDO URICO Y CALCIURIA. DIFERENTES VARIABLES

SEGUN DIAGNOSTICO

\begin{tabular}{|lcccc|}
\hline & $\begin{array}{c}\text { n=75 } \\
\text { H.I.E. }\end{array}$ & H.A.C. & $\begin{array}{c}\text { n= 36 } \\
\text { H.T.G. }\end{array}$ & $\begin{array}{c}\mathbf{n}=\mathbf{8} \\
\text { H.A.S. }\end{array}$ \\
\hline Edad & $24,37 \pm 6,5$ & $33,87 \pm 7,9$ & $25,72 \pm 6,7$ & $31,87 \pm 6,9$ \\
Paridad & $0,7 \pm 1,3$ & $3,1 \pm 2,5$ & $1,1 \pm 1,6$ & $3,3 \pm 2,7$ \\
$\begin{array}{l}\text { Edad gestacional } \\
\text { semanas) }\end{array}$ & $37,0 \pm 4,0$ & $35,7 \pm 3,2$ & $38,9 \pm 1,7$ & $35,8 \pm 3,8$ \\
$\begin{array}{l}\text { Tensión arterial } \\
\text { Sistólica mm/Hg }\end{array}$ & $154,6 \pm 17$ & $161,2 \pm 14,5$ & $144,3 \pm 12$ & $171,2 \pm 29,8$ \\
$\begin{array}{l}\text { Tensión arterial } \\
\text { diastólica mm/Hg }\end{array}$ & $102,4 \pm 9,2$ & $103,7 \pm 5,1$ & $97,8 \pm 6,6$ & $109,7 \pm 13$ \\
$\begin{array}{l}\text { Tensión arterial } \\
\text { media }\end{array}$ & $119 \pm 10,2$ & $120,7 \pm 8,5$ & $113,2 \pm 9,0$ & $129,1 \pm 17,8$ \\
\hline
\end{tabular}

Con respecto a los síntomas reportados por la paciente y los signos hallados al examen clínico, el edema es el más frecuente seguido por la hiperreflexia y la cefalea (tabla 2). La tabla 3 permite observar los valores para las diferentes determinaciones de laboratorio según diagnóstico. Con relación a la forma de terminación del embarazo, se realizó cesárea en 58 de ellas (43\%), parto instrumentado en siete $(5,2 \%)$ y parto normal en $70(51,9 \%)$. Sólo se presentó una muerte materna con diagnóstico de Síndrome de Hellp y edema agudo de pulmón, registrándose 10 muertes perinatales en el grupo.
Tabla 2

SIGNOS Y SINTOMAS PRESENTADOS POR LAS PACIENTES, EN ORDEN DE FRECUENCIA

\begin{tabular}{|lc|}
\hline Signos y síntomas & $\%$ \\
\hline - Edema & $60,6 \%$ \\
- Hiperreflexia & $46,2 \%$ \\
- Cefalea & $38,3 \%$ \\
- Epigastralgia & $25,6 \%$ \\
- Fosfenos & $21,1 \%$ \\
- Trastornos de conciencia & $5,3 \%$ \\
\hline
\end{tabular}


ACIDO URICO Y CALCIURIA. OTRAS DETERMINACIONES DE LABORATORIO

\begin{tabular}{|lccccc|}
\hline Diagnóstico & H.I.E. & H.A.C. & H.T.G. & H.A.S. & $\begin{array}{c}\text { Valores } \\
\text { normales }\end{array}$ \\
\hline Examen & & & & & \\
\hline Proteinuria & & & & & \\
orina 24 horas & 2,96 & 0,125 & 0,129 & 7,84 & $350 \mathrm{mg} / 24 \mathrm{~h}$ \\
g/litro & $\pm 5,0$ & $\pm 0,05$ & $\pm 0,07$ & $\pm 12,39$ & \\
Creatinina & 0,8 & 0,83 & 0,77 & 0,94 & $<0$ \\
mg \% & $\pm 0,27$ & $\pm 0,18$ & $\pm 0,17$ & $\pm 0,24$ & $<15$ \\
Nitrógeno & 9,2 & 8,14 & 7,94 & 12,06 & \\
úrico & $\pm 3,9$ & $\pm 1,3$ & $\pm 3,27$ & $\pm 5,01$ & $150.000-$ \\
Plaquetas & 254.166 & 262.875 & 257.666 & 205.000 & 300.000 \\
\hline
\end{tabular}

Los valores de calciuria se obtuvieron en 101 de las pacientes y los de ácido úrico en 119 de ellas. Se observó una disminución estadísticamente significativa $(\mathrm{p}<0,01)$ de los niveles de calciuria en orina de 24 horas en las pacientes con: hipertensión inducida por el embarazo $(195,13 \pm 124,6 \mathrm{mg} / 24$ horas $)$, hipertensión arterial crónica + preeclampsia sobreagregada $(160,23 \pm 79,3 \mathrm{mg} / 24$ horas) y en menor proporción en las pacientes con hipertensión transitoria $(233,7 \pm 141,9 \mathrm{mg} / 24$ horas $)$. Por el contrario, los niveles de calciuria aumentaron ligeramente en pacientes con hipertensión arterial crónica. Se hallaron elevaciones significativas del ácido úrico en todas las pacientes con trastornos hipertensivos de la gestación. Tabla 4.

Tabla 4

VALORES DE ACIDO URICO Y CALCIURIA

\begin{tabular}{|lcccc}
\hline Diagnóstico & $\begin{array}{c}\text { Acido úrico } \\
\mathbf{m g} / \mathbf{d l}\end{array}$ & P* & $\begin{array}{c}\text { Calciuria } \\
\mathbf{~ m g / 2 4} \text { horas }\end{array}$ & P** \\
\hline $\begin{array}{l}\text { Preeclampsia } \\
\text { Eclampsia }\end{array}$ & $5,55 \pm 1,55$ & $<0,01$ & $195,13 \pm 124,6$ & $<0,01$ \\
$\begin{array}{l}\text { Hipertensión } \\
\text { arterial crónica }\end{array}$ & $5,51 \pm 1,91$ & $<0,01$ & $334,25 \pm 305,9$ & $* *$ \\
$\begin{array}{l}\text { Hipertensión } \\
\text { crónica + preeclampsia } \\
\text { sobreagregada } \\
\begin{array}{l}\text { Hipertensión } \\
\text { transitoria }\end{array}\end{array}$ & $5,90 \pm 1,69$ & $<0,01$ & $160,23 \pm 79,3$ & $<0,01$ \\
$\begin{array}{l}\text { Embarazo normal } \\
\text { (valor de referencia) }\end{array}$ & $5,0 \pm 1,65$ & $<0,01$ & $233,7 \pm 141,9$ & $<0,01$ \\
\hline
\end{tabular}

Los datos se expresan como promedio y desvío estándar.

* $\quad$ Prueba de $\mathrm{Z}$ (nivel de significancia $\mathrm{P}<0,01$ ).

** Los valores de calciuria en el grupo de pacientes con hipertensión crónica son significativamente más altos que el promedio. 


\section{Discusión}

En el presente estudio se halla una disminución significativa de los niveles de calciuria en orina de 24 horas en pacientes con preeclampsia eclampsia, hipertensión arterial crónica más preeclampsia sobreagregada y en menor proporción en pacientes con hipertensión transitoria gestacional. No se observó disminución de los niveles de calciuria en las pacientes con hipertensión arterial crónica. Figura 1.

Esta disminución de los niveles de calciuria ha sido reportada por otros autores, postulándose como posible mecanismo de la hipocalciuria al incremento de la reabsorción tubular de calcio en este tipo de alteraciones. $(2,4,6,8-9,26)$.

Dentro de los fenómenos hipertensivos de la gestación se considera como el de peor pronóstico a la hipertensión crónica + preeclampsia sobreagregada en razón a las complicaciones maternas y fetales asociadas. La posibilidad de detectar tempranamente la aparición de preeclampsia en este grupo de pacientes mediante la determinación periódica de los niveles de calciuria, puede convertirse en una metodología diagnóstica eficaz, permitiendo tomar decisiones oportunas. La elevación de los niveles de ácido úrico puede considerarse también un indicador de la existencia de la patología, aunque su capacidad discriminatoria de los diferentes tipos de trastorno no parece tan confiable si se tiene en cuenta el aumento de los niveles de ácido úrico registrado en los cuatro grupos de pacientes. Figura 2.

Además de las determinaciones de laboratorio rutinarias, se propone la estimación de la calciuria en orina de 24 horas como método diagnóstico y de tamizaje de los trastornos hipertensivos de la gestación, en particular la preeclampsia - eclampsia y la hipertensión arterial crónica con preeclampsia sobreagregada.

\section{Agradecimientos}

A todos los miembros del Departamento de Ginecología y Obstetricia del Hospital de Caldas que en alguna forma colaboraron en el trabajo.
Al personal del Laboratorio Clínico del Hospital.

A los doctores Carlos Alberto Esparza Duque, profesor del Departamento de Gineco-obstetricia, José Arnoby Chacón y Eduardo León Jaramillo, por sus valiosas orientaciones.

\section{Figura 1}

ACIDO URICO Y CALCIURIA. VALOR PRONOSTICO Y DIAGNOSTICO

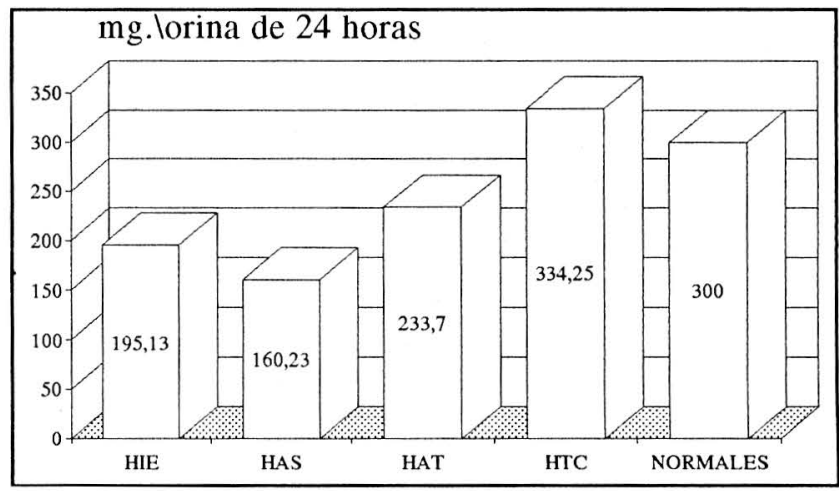

Figura 2

ACIDO URICO Y CALCIURIA. VALOR PRONOSTICO Y DIAGNOSTICO

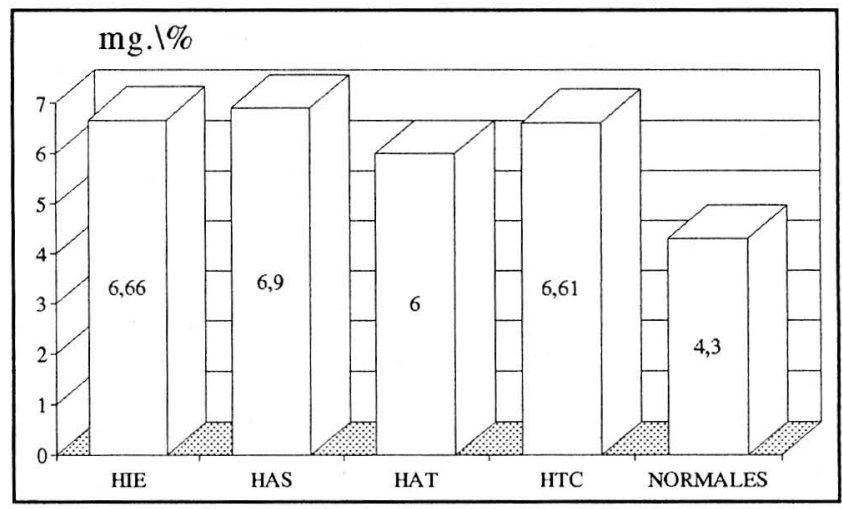

\section{BIBLIOGRAFIA}

1. Vélez J., Dávila L. Mortalidad materna institucional en Caldas: 19901995.

2. Masse J., Forest JC., Moutquin J. A prospective study of several potential biologic markers for early prediction of the development of preeclampsia. Am. J. Obstet. Gynecol. 1993; 169: 501-508.

3. López P. y de Félix M. Uso del calcio urinario en la prevención de la hipertensión inducida por el embarazo. Boletín de la Oficina Sanitaria Panamericana. $1991 ; 110(2): 126-134$.

4. Sánchez RL., Jones OC., Cullen MJ. Urinary calcium as an early marker for preeclampsia. Obstet. Gynecol. 1991; 77:685-688.

5. Belizan J. y Villar J. The relation ship between calcium intake and edema-proteinuria, and hypertension-gestosis: an hypothesis. Am. J. Clin. Nutr. 1980; 33: 2202-2210.

6. Rodríguez MH., Masaki RJ., Mestman J., Kuman Omesky y Rude R. Calcium/creatinine ratio and microalbuminuria in the prediction of preeclampsia. Am. J. Obstet. Gynecol. 1988; 159:1452-1455.
7. Villar J., Repne J., Belizan J. y Pareja G. Calcium suplementation reduces blood pressure during pregnancy: results of a randomized controlled clinical trial. Obstet. Gynecol. 1987; 70 (317): 317-321.

8. Taufield P., Ales K., Rejnick L. Hypocalciuria in preeclampsia. N. Eng. Med; 1987; 316: 715-718.

9. Sánchez Ramos L., Sandroni S., AndrésFJ. and Kaunitz AM. Calcium excretion in preeclampsia. Obstetrics and Gynecology. 1991; 77: 510-513.

10. Pitkin R. Calcium metabolism in pregnancy and the perinatal period: a review. Am. J. Obstet. Gynecol. 1985; 151:99-109.

11. Belizan JM., Villar J., Salazar A. y cols. Evidencia nutricional del efecto de suplementación de calcio en la presión sanguínea en mujeres embarazadas normales. Am. J. Obstet. Gynecol. 146, $\mathbf{N}^{\circ} 2,1980$.

12. O’Brien W. Pronóstico de la preeclampsia. Clínicas Obstétricas y Ginecológicas 2, 1992; 341-350.

13. Balcells, A. La clínica y el laboratorio. 14 ed. Barcelona: Marín. $1986 ; 597$. 
14. Schwarcz R., Durerges CA., Díaz AG., Fescina R. Obstetricia. 4 ed. Buenos Aires: El Ateneo. 1988; 220.

15. Botero J., Jubiz A., Henao G. Obstetricia y Ginecología. 3 ed. Medellín: Carvajal, 1987; 273.

16. Zuspan F. New concepts in the understanding of hypertensive diseases during pregnancy. Clinics in perinatology. 1991; 18(4): 653-659.

17. Burrow G., Ferris T. Complicaciones médicas durante el embarazo. $2^{\mathbf{a}}$ ed. Buenos Aires: Médica Panamericana, 1984.

18. Sibai B. Hipertension and pregnancy clinicos in perinatology. 1971; 18(4).

19. Polit D., Bernadette H. Investigación científica en ciencias de la salud. $2^{a}$ ed. México D.F.: Interamericana, 1987.

20. Belitzky R., Nieto F., Martell M., Fescine R. Metodología para la investigación epidemiológica operacional y clínica con ejemplos perinatales. Centro Latinoamericano de Perinatología y Desarrollo Humano. O.P.S. Montevideo, Uruguay, 1985.

21. Dildy G., Colton D. Management of severe preeclampsia and eclampsia. In: Carlson R., Geheb M. Obstetric emergencies. Critical care clinics. $1991 ; 7(4): 829-849$.
22. Colton. Stadistics in medicine. Boston: Little Brown, 1984.

23. Sibai BM., Anderson GD., McCubbib JH. Eclampsia II. Clinical significance of laboratory findings. Obstet. Gynecol, 1982; 59:153157.

24. Gant N., Pritchard J. Pregnancy-induced hypertension. In: Eden R., Boehm F. Assessment and care of the fetus. Physiological, clinical and medico legal principles. Appleton \& Lange, 1990; 711-723.

25. Yoshimura A., Ideura T., Iwasaki S. Significance of uric acid clearance in preeclampsia. In: Am. J. of Obstet. and Gynecol., 1990; 162:16391670. (Letters).

26. Rodríguez K. y Calero R. El síndrome preeclampsia-eclampsia. Revista Colombiana de Obstetricia y Ginecología. 1993; 44(4):267271.

27. Dekker G., Sibai B. Early detection of preeclampsia. Am. J. Obstet. Gynecol., 1991; 165:160-172.

28. Arias F. Guía práctica para el embarazo y el parto de alto riesgo. $2^{\mathrm{a}}$ ed. Madrid: Mosby/ Doyma Libros. 1994; 190.

\section{EVENTOS}

\section{CONGRESO COLOMBIA NO DE OBSTETRICIA Y GINECOLOCIA}

Hotel Intercontinental - Medellín

\section{Informes:}

Asociación Antioqueña de Obstetricia y Ginecología

Tel. (942) 1640.11 y en Santa Fe de Bogotá 2699903

\section{CONGRESO CENTROAMERICANO DE OBSTETRICIA Y GINECOLOGIA}

\section{San Sal vador}

13 al 17 de noviembre de 1995

\section{MORTALIDAD MATERNA \\ Hotel Ramada Renaissance Jaragua Santo Domingo \\ 12 al 16 de marzo de 1996 \\ lintormes: \\ Tel. (809) 682-2475. Santo Domingo}

\section{FE DE ERRATAS}

En el Sumario del Nümero 2, Volumen 46 de la Revista Colombiana de Obsterricia y Ginecología, los articulos contenidos entre las páginas 95 y 129 son Contribuciones. 